\title{
On Harmonious Colouring of Trees
}

\author{
A. Aflaki ${ }^{a}$, S. AKBARI ${ }^{\mathrm{a}, \mathrm{b}}$, K.J. EdWARDS ${ }^{\mathrm{c}}$, \\ D.S. EskANDANi ${ }^{a}$, M. JAMAALI ${ }^{a}$, H. RAVANBOD ${ }^{a}$, \\ a Department of Mathematical Sciences, Sharif University of Technology, Tehran, Iran \\ ${ }^{\mathrm{b}}$ School of Mathematics, Institute for Research in Fundamental Sciences (IPM), Tehran, Iran \\ P.O. Box 19395-5746, Tehran, Iran \\ ${ }^{\mathrm{c}}$ School of Computing, University of Dundee, Dundee DD1 4HN, Scotland *
}

Submitted: Apr 1, 2011; Accepted: Dec 17, 2011; Published: Jan 6, 2012

Mathematics Subject Classifications: 05C05, 05C15

\begin{abstract}
Let $G$ be a simple graph and $\Delta(G)$ denote the maximum degree of $G$. A harmonious colouring of $G$ is a proper vertex colouring such that each pair of colours appears together on at most one edge. The harmonious chromatic number $h(G)$ is the least number of colours in such a colouring. In this paper it is shown that if $T$ is a tree of order $n$ and $\Delta(T) \geqslant \frac{n}{2}$, then there exists a harmonious colouring of $T$ with $\Delta(T)+1$ colours such that every colour is used at most twice. Thus $h(T)=\Delta(T)+1$. Moreover, we prove that if $T$ is a tree of order $n$ and $\Delta(T) \leqslant\left\lceil\frac{n}{2}\right\rceil$, then there exists a harmonious colouring of $T$ with $\left\lceil\frac{n}{2}\right\rceil+1$ colours such that every colour is used at most twice. Thus $h(T) \leqslant\left\lceil\frac{n}{2}\right\rceil+1$.
\end{abstract}

\section{Introduction}

All graphs considered in this paper are finite, undirected, with no loops or multiple edges. Let $G$ be a graph. We denote the edge set and the vertex set of $G$ by $E(G)$ and $V(G)$, respectively. A vertex of degree 1 in $G$ is called a pendant vertex. The number of vertices of $G$ is called the order of $G$. We denote the maximum degree of $G$ by $\Delta(G)$ and for simplicity by $\Delta$. Also, for every $v \in V(G)$ and $X \subseteq V(G), d(v), N_{G}(v)$ and $N_{G}(X)$ denote the degree of $v$, the neighbor set of $v$ and the set of vertices of $G$ which have at least one neighbor in $X$, respectively. For simplicity we use $N(v)$ and $N(X)$ instead of $N_{G}(v)$ and $N_{G}(X)$, respectively. Also, we use $N_{G}[v]$ for $N_{G}(v) \cup\{v\}$. By a unicyclic graph, we mean a connected graph with exactly one cycle. For a natural number $k$, a graph $G$

\footnotetext{
*E-mail addresses: arian.aflaki@gmail.com, s_akbari@sharif.edu, kjedwards@dundee.ac.uk denizeskandani@gmail.com,mjamaali@sharif.edu, hajar_ravanbod@yahoo.com.
} 
is called a $k$-regular graph if $d(v)=k$, for each $v \in V(G)$. A projective plane of order $q$ consists of a set $X$ of $q^{2}+q+1$ elements called points, a family $\mathcal{L}$ of subsets called lines, having the following properties:

(i) Every line has $q+1$ points.

(ii) Every point lies on $q+1$ lines.

(iii) Any two points lie on a unique line.

We denote the projective plane of order $q$ by $\mathrm{PG}(q)$. It is well known that if $q$ is a prime power, then $\mathrm{PG}(q)$ exists.

Let $G$ be a graph. A proper vertex colouring of $G$ is a function $c: V(G) \longrightarrow L$, such that if $u, v \in V(G)$ are adjacent, then $c(u)$ and $c(v)$ are different. A proper vertex $k$-colouring is a proper vertex colouring with $|L|=k$. A harmonious colouring of $G$ is a proper vertex colouring of $G$ in which every pair of colours appears on at most one pair of adjacent vertices. The harmonious chromatic number of $G, h(G)$, is the minimum number of colours needed for any harmonious colouring of $G$. The first paper on harmonious colouring was written in 1982 by Frank et al. [4]. However, the usual definition of this notion is due to Hopcroft and Krishnamoorthy [5]. The concept of harmonious colouring of graphs has been studied extensively by several authors; see $[2,7]$ for surveys. If $G$ has $m$ edges and $G$ has a harmonious colouring with $k$ colours, then clearly, $\left(\begin{array}{l}k \\ 2\end{array}\right) \geqslant m$.

Let $k(G)$ be the smallest integer satisfying the inequality. This number can be expressed as a function of $m$, namely

$$
k(G)=\left\lceil\frac{1+\sqrt{8 m+1}}{2}\right\rceil .
$$

Paths are among the first graphs whose harmonious chromatic numbers have been established. Let $P_{n}$ denote the path of order $n$. The following fact has been proved [4].

If $k\left(P_{n}\right)$ is odd or if $k\left(P_{n}\right)$ is even and $n-1=k(k-1) / 2-j, j=k / 2-1, k / 2, \ldots, k-2$, where $k=k\left(P_{n}\right)$, then $h\left(P_{n}\right)=k\left(P_{n}\right)$. Otherwise, $h\left(P_{n}\right)=k\left(P_{n}\right)+1$.

It was shown by Hopcroft and Krishnamoorthy that the problem of determining the harmonious chromatic number of a graph is NP-hard. Also, it was shown that the problem remains hard even when we restricted to trees, see [3]. The following result has been proved in $[1]$.

Let $d$ be a fixed positive integer. There is a positive integer $N$ such that if $T$ is any tree with $m \geqslant N$ edges and maximum degree at most $d$, then $h(T)$ is either $k(G)$ or $k(G)+1$.

In this paper we obtain the exact value of the harmonious chromatic number of a tree when its maximum degree is at least the half of its order.

The harmonious edge colouring of a graph is a proper edge colouring in which every pair of colours appears on at most one pair of adjacent edges. The harmonious edge chromatic number of $G, h^{\prime}(G)$, is the minimum number of colours needed for a harmonious edge colouring of $G$. The line graph of $G, L(G)$, is a graph that has a vertex for every edge of $G$, and two vertices of $L(G)$ are adjacent if and only if the corresponding two edges in $G$ are adjacent. Clearly, for every graph $G, h^{\prime}(G)=h(L(G))$. 


\section{Harmonious Colouring of Trees}

In this section we wish to find a harmonious colouring for a tree with the property that each colour is used at most twice. Also, we show that for every bipartite graph $G$ of order $n, h(G) \leqslant \frac{n}{2}+\Delta^{2}-\Delta$, where $\Delta$ is the maximum degree of $G$.

Theorem 1. Let $T$ be a tree of order $n$ and $\Delta \geqslant \frac{n}{2}$. Then there exists a harmonious colouring of $T$ with $\Delta+1$ colours such that every colour is used at most twice. Thus $h(T)=\Delta+1$.

Proof. Let $v$ be a vertex of maximum degree and $N(v)=\left\{v_{1}, \ldots, v_{\Delta}\right\}$. Assume that $G_{1}, \ldots, G_{\Delta}$ are connected components of $T \backslash v$ such that $v_{i} \in V\left(G_{i}\right)$, for $i=1, \ldots, \Delta$. With no loss of generality assume that $\left|V\left(G_{1}\right)\right| \geqslant \cdots \geqslant\left|V\left(G_{k}\right)\right| \geqslant 2$ and $\left|V\left(G_{i}\right)\right|=1$, for $i=k+1, \ldots, \Delta$. We want to define a harmonious colouring $c$ of $T$. First, define $c\left(v_{i}\right)=i$, for $i=1, \ldots, \Delta$ and $c(v)=\Delta+1$. Let $n_{i}=\left|V\left(G_{i}\right)\right|$, for $i=1, \ldots, k$. Colour the vertices of $G_{1} \backslash v_{1}$, by the colours $2, \ldots, n_{1}$ such that each colour is used once. Colour the vertices of $G_{2} \backslash v_{2}$ by the colours $n_{1}+1, \ldots, n_{1}+n_{2}-1$ such that each colour is used once. Repeat this procedure and colour the vertices of $G_{k} \backslash v_{k}$ by the colours $n-(\Delta+1)-n_{k}+3, \ldots, n-(\Delta+1)+1$ such that each colour is used once. It is straightforward to check that this colouring is a harmonious colouring of $G$. Hence $h(T) \leqslant \Delta+1$. Clearly, in each harmonious colouring of $G$ the neighbors of each vertex should get different colours. So $h(T)=\Delta+1$ and the proof is complete.

Lemma 2. Let $T$ be a tree with bipartition $(A, B)$, where $|A|=|B|=n / 2$. Then $T$ has a harmonious colouring with at most $n / 2+1$ colours, such that each colour occurs at most once in each part of the bipartition.

Proof. Let $r=n / 2$. We use induction on $r$. If $r=1$, then $T=K_{2}$ and the result is obvious. So suppose that $r>1$. Note that each of $A$ and $B$ has $n-1$ incident edges and $n / 2$ vertices, and so has average degree strictly less than 2 . Hence we can assume that there are leaves $a \in A$ and $b \in B$. Delete $a$ and $b$ to form a tree $T^{\prime}$ with bipartition $A^{\prime}, B^{\prime}$ where $\left|A^{\prime}\right|=\left|B^{\prime}\right|=r-1$. By the inductive hypothesis, there is a harmonious colouring $c$ of $T^{\prime}$ with colours $1, \ldots, r$ such that each colour occurs at most once on $A^{\prime}$ and at most once on $B^{\prime}$. Let $u \in B, v \in A$ be the neighbours in $T$ of $a, b$ respectively. If $c(u) \neq c(v)$, then we obtain the desired colouring of $T$ by colouring $a$ and $b$ with a new colour $r+1$. If $c(u)=c(v)=i$, then let $j, k$ be the colours from $1, \ldots, r$ not used on $A, B$ respectively. Recolour $u$ with colour $r+1$, and set $c(a)=j, c(b)=k$. It is clear that this gives the desired colouring of $T$.

Theorem 3. Let $T$ be a tree with $n$ vertices and with bipartition $(A, B)$, where $|A| \leqslant|B|$. Suppose that $T$ has maximum degree at most $\lceil n / 2\rceil$. Then $T$ has a harmonious colouring with at most $\lceil n / 2\rceil+1$ colours, such that each colour occurs at most twice, and each vertex of $A$ has a distinct colour. 
Proof. If $|A|=|B|$, then the result follows immediately from Lemma 2. Otherwise, let $x=|B|-|A|$, and let $t$ be the number of leaves of $B$. Then the number of edges incident with $B$ is at least $t+2(|B|-t)=2|B|-t$, and so $n-1 \geqslant 2|B|-t$. Then since $n=|A|+|B|=2|B|-x$, we have $2|B|-x-1 \geqslant 2|B|-t$, so that $t>x$. Hence we can find a set $X$ of leaves in $B$ with $|X|=x$. Delete the set $X$ of leaves to form a tree $T^{\prime}$. Then $T^{\prime}$ has bipartition $(A, B \backslash X)$ where $|A|=|B \backslash X|$.

Hence by Lemma $2, T^{\prime}$ has a harmonious colouring with a set $S$ of at most $|A|+1$ colours so that each colour occurs at most once on $A$ and at most once on $B \backslash X$. Note that $n=2|A|+x$, so that $\lceil n / 2\rceil+1=\lceil x / 2\rceil+|A|+1$. Thus we require to find a colouring of $T$ using at most $\lceil x / 2\rceil$ extra colours.

First, if two uncoloured leaves in $X$ have distinct neighbours (which therefore have distinct colours), then colour these two leaves with a new colour. We can repeat this as long as there are two uncoloured leaves in $X$ with distinct neighbours. If all vertices in $X$ are coloured by the process, then we have the required colouring. Otherwise, we are left with a set $Y$ of uncoloured leaves, which are all neighbours of the same vertex $v \in A$. Let $y=|Y|$. Since each new colour has been used for two vertices, and $x-y$ is even, then we have now used at most $|A|+1+(x-y) / 2=|A|+1+\lceil x / 2\rceil-\lceil y / 2\rceil$ colours. Hence there are at least $\lceil y / 2\rceil$ colours still unused. Let $j$ be the colour which does not occure on $B \backslash X$ in the colouring of $T^{\prime}$. If $j \neq c(v)$, then there is a vertex $u$ in $B \backslash X$ which has colour $c(v)$. In that case, recolour $u$ with a new colour $i$, and colour two elements $y_{1}, y_{2}$ of $Y$ with colours $i, j$ respectively (or if $y=1$, then colour the single vertex with colour $i$ ). If every vertex is now coloured, then the proof is complete, otherwise let $Z$ be the set of remaining uncoloured leaves, and let $z=|Z|$. Note that if $j \neq c(v)$, then $Z=Y \backslash\left\{y_{1}, y_{2}\right\}$, otherwise $Z=Y$. In either case, we have a set $N$ of $\lceil z / 2\rceil$ colours still unused.

The number of colours which occur adjacent to $c(v)$ is $d(v)-z$, hence the number of colours (apart from $c(v)$ itself) not used adjacent to $c(v)$ is at least $\lceil n / 2\rceil-d(v)+z$. Since $d(v) \leqslant\lceil n / 2\rceil$, this is at least $z$. Hence, in addition to those in $N$ we can find a set $S$ of $\lfloor z / 2\rfloor$ colours (with $c(v) \notin S$ ) which do not occur adjacent to $c(v)$. The colours in $S$ may occur (possibly twice) on $B$; recolour one vertex of each such colour using at most $\lfloor z / 2\rfloor$ colours from $N$. Now each colour in $N \cup S$ occurs at most once, and does not occur adjacent to $c(v)$. Hence these colours can be used on the remaining $z$ leaves; this completes the proof.

Theorem 4. If $T$ is a tree, then $h(T) \leqslant|B|+\Delta$, where $T=(A, B)$ is a bipartition of $T$ and $|B| \leqslant|A|$.

Proof. We show that there is a harmonious colouring using $|B|+\Delta$ colours. One can find a labeling for the vertices of $T$, say $\left\{v_{1}, \ldots, v_{n}\right\}$, such that for every $i, i>1$, there exists a unique vertex $v_{j}, j<i$, such that $v_{i}$ is adjacent to $v_{j}$. Let $i$ be the smallest index such that $v_{i}$ has no colour and the harmonious property holds for every coloured vertex. We want to colour $v_{i}$ so that the harmonious property holds. By assumption there exists at most one vertex $v_{j}, j<i$, such that $v_{j}$ has been coloured. The vertex $v_{j}$ has at most $\Delta$ neighbors in $A$. Since we have $\Delta$ colours which are not used in the vertex colouring of $B$, there is at least one colour which is not used in the neighbor of $v_{j}$ among $\Delta$ new colours, 
say $\alpha$. Colour $v_{i}$ by $\alpha$. Suppose that the colour of $v_{j}$ is $\beta$. If there exists a vertex $v_{k} \in B$ such that $v_{k}$ is adjacent to a vertex $v_{t} \in A$ with colour $\alpha$, and $v_{k} \in N\left(v_{i}\right)$, then noting to the labeling of vertices, we have $k>i$. On the other hand $i>t$. Thus $v_{k}$ is adjacent to two vertices with indices smaller than $k$, a contradiction. The proof is complete.

In the following theorem we show that for every real number $\alpha, 0 \leqslant \alpha<1$, there exists a bipartite graph $G$ such that $h(G)>\alpha n+\Delta$, where $n=|V(G)|$.

Theorem 5. For bipartite graph $G$, the inequality $h(G) \leqslant \frac{n}{2}+\Delta$ is not necessarily true.

Proof. Let $k$ be a natural number and $\mathrm{PG}(k)$ be the projective plane of order $k$. Assume that $m$ is a natural number such that $\frac{m}{m+1}>\alpha$. We form a bipartite graph $G=(A, B)$, where $A$ contains $m$ copies of each point and $B$ contains a vertex corresponding to each line of $\mathrm{PG}(k)$. Thus $|A|=m\left(k^{2}+k+1\right)$ and $|B|=k^{2}+k+1$. Join point $p \in A$ to line $L \in B$ if $p \in L$.

We have $|V(G)|=(m+1)\left(k^{2}+k+1\right)$ and $\Delta=m(k+1)$. Since for a pair of points there is a line containing the pair, then in each harmonious colouring of $G$, the vertices of $A$ must have different colours. This implies that $h(G) \geqslant m\left(k^{2}+k+1\right)$. We have $\alpha|V(G)|+\Delta=$ $\alpha(m+1)\left(k^{2}+k+1\right)+m(k+1)$. If $k$ is sufficiently large, then $\alpha|V(G)|+\Delta<m\left(k^{2}+k+1\right)$. Thus $h(G)>\alpha|V(G)|+\Delta$.

Theorem 6. Let $G=(A, B)$ be a bipartite graph, $\Delta \geqslant 2$ and $|B| \leqslant|A|$. Then $h(G) \leqslant$ $|B|+\Delta^{2}-\Delta$.

Proof. Assume that $A=\left\{v_{1}, \ldots v_{|A|}\right\}$ and $B=\left\{u_{1}, \ldots, u_{|B|}\right\}$ are two parts of the graph $G$. We colour the vertices of $B$ with $|B|$ different colours. Now, we colour the vertices of Part $A$ step by step. First, we want to colour Part $A$ using $\Delta^{2}-\Delta+1$ new colours. Colour vertex $v_{1}$ by one of the arbitrary colours among $\Delta^{2}-\Delta+1$ new colours. Now, assume that $v_{1}, \ldots, v_{i}$ have been coloured such that the induced subgraph on the vertices $u_{1}, \ldots, u_{|B|}, v_{1}, \ldots, v_{i}$, has a harmonious colouring. Now, we try to colour $v_{i+1}$. Let $N\left(v_{i+1}\right)=\left\{u_{i_{1}}, \ldots, u_{i_{k}}\right\}$, for some $k$. For every $j, 1 \leqslant j \leqslant k, u_{i_{j}}$ has at most $\Delta-1$ coloured neighbors. Thus $N\left(N\left(v_{i+1}\right)\right)$ has at most $k(\Delta-1) \leqslant \Delta(\Delta-1)$ coloured neighbors. But we have $\Delta^{2}-\Delta+1$ colours which are not used in the colouring of $B$. This shows that we have at least one available colour for the colouring of $v_{i+1}$ such that the induced subgraph on $u_{1}, \ldots, u_{|B|}, v_{1}, \ldots, v_{i+1}$, has a harmonious colouring.

Now, we would like to reduce the number of colours by 1 . If in the colouring of $A$ we use at most $|B|+\Delta^{2}-\Delta$ colours, then we are done. Assume that $u \in B$. Since $\Delta^{2}-\Delta+1>\Delta$, then there exists at least one colour, say $\alpha$, among $\Delta^{2}-\Delta+1$ new colours which is not used in the colouring of the neighbors of $u$. Now, recolour the vertex $u$ by $\alpha$ to obtain a harmonious colouring of $G$ by $|B|+\Delta^{2}-\Delta$ colours.

Corollary 7. Let $G$ be a bipartite graph of order $n$. Then $h(G) \leqslant \frac{n}{2}+\Delta^{2}-\Delta$. 


\section{Harmonious Edge Colouring of Graphs}

In this section we extend the concept of harmonious colouring to harmonious edge colouring. By the definition clearly for every graph $G, h^{\prime}(G)=h(L(G))$, where $L(G)$ is the line graph of $G$.

Theorem 8. Let $G$ be a connected graph. If $G$ is a tree, then $h(G) \leqslant h^{\prime}(G)+1$ and if $G$ is a unicyclic graph, then $h(G) \leqslant h^{\prime}(G)$.

Proof. Let $c^{\prime}: E(G) \rightarrow\left\{1, \ldots, h^{\prime}(G)\right\}$ be a harmonious edge colouring of $G$. Now, using $c^{\prime}$ we wish to obtain a harmonious colouring of $G$, say $c: V(G) \rightarrow\left\{1, \ldots, h^{\prime}(G)+1\right\}$. Assume that $L_{1}=\left\{v \in V(G) \mid d_{G}(v)=1\right\}$. For every $v \in L_{1}$, define $c(v)=c^{\prime}\left(e_{v}\right)$, where $v$ and edge $e_{v}$ are incident. Now, let $G_{1}=G \backslash L_{1}$. Suppose that

$$
L_{2}=\left\{v \in V\left(G_{1}\right) \mid d_{G_{1}}(v)=1\right\} .
$$

For every $v \in L_{2}$, define $c(v)=c^{\prime}\left(e_{v}\right)$, where $v$ and $e_{v}$ are incident in $G_{1}$. Let $G_{2}=G_{1} \backslash L_{2}$. Now, inductively $G_{i}$ and $L_{i}$ are defined as follows:

$$
L_{i+1}=\left\{v \in V\left(G_{i}\right) \mid d_{G_{i}}(v)=1\right\}
$$

and $G_{i+1}=G_{i} \backslash L_{i+1}$, where $G_{0}=G$. For each $v \in L_{i+1}$, define $c(v)=c^{\prime}\left(e_{v}\right)$, where $v$ and $e_{v}$ are incident. Assume that $r$ is the minimum number such that $L_{r+1}=\emptyset$. Now, since $G$ is a connected graph, two cases can be considered:

Case 1. $G$ is a tree. Thus $G_{r}$ is an isolated vertex. Let $V\left(G_{r}\right)=\{w\}$. Now, define $c(w)=h^{\prime}(G)+1$. We claim that $c$ is a harmonious colouring of $G$. First we show that $c$ is a proper colouring. Assume that $c(u)=c(v)$ and $u v \in E(G)$. Thus $c^{\prime}\left(e_{u}\right)=c^{\prime}\left(e_{v}\right)$. Clearly, $u v \in\left\{e_{u}, e_{v}\right\}$. With no loss of generality assume that $u v=e_{u}$. Since $u v$ and $e_{v}$ are adjacent and they have the same colour, this contradicts the properness of $c^{\prime}$. Hence $c$ is a proper colouring of $G$. Next, we prove that $c$ is a harmonious colouring. Let $\{c(u), c(v)\}=\{c(x), c(y)\}, u v, x y \in E(G)$, and $u v \neq x y$. With no loss of generality assume that $c(u)=c(x)$ and $c(v)=c(y)$. Therefore $c^{\prime}\left(e_{u}\right)=c^{\prime}\left(e_{x}\right)$ and $c^{\prime}\left(e_{v}\right)=c^{\prime}\left(e_{y}\right)$. Since $u v \in\left\{e_{u}, e_{v}\right\}$ and $x y \in\left\{e_{x}, e_{y}\right\}$, one may assume that $u v=e_{u}, x y=e_{x}$. Now, since $x y$ and $e_{y}$, and $u v$ and $e_{v}$, respectively are incident and $\left\{c^{\prime}(x y), c^{\prime}\left(e_{y}\right)\right\}=\left\{c^{\prime}(u v), c^{\prime}\left(e_{v}\right)\right\}$, we conclude that $c^{\prime}$ is not a harmonious edge colouring, a contradiction.

Case 2. $G$ is a unicyclic graph. Thus $G_{r}=C_{k}$, for some $k \geqslant 3$. Assume that $C_{k}$ is as follows:

$$
v_{1} e_{1} v_{2} e_{2} \ldots v_{k-1} e_{k-1} v_{k} e_{k} v_{1}
$$

For every $i, 1 \leqslant i \leqslant k$ define $c\left(v_{i}\right)=c^{\prime}\left(e_{i}\right)$. Similar to the Case 1 , it is not hard to see that this colouring is a harmonious colouring of $G$.

Now, we propose the following conjecture.

Conjecture. If $G$ is a connected graph and $G$ is not a tree, then $h(G) \leqslant h^{\prime}(G)$. 
Remark. It is easily seen that if $G$ is a strongly regular graph with positive parameters, then $h(G)=|V(G)|$. Thus $h^{\prime}\left(K_{n}\right)-h\left(K_{n}\right)=\left(\begin{array}{l}n \\ 2\end{array}\right)-n$. So the difference between $h^{\prime}(G)$ and $h(G)$ can be arbitrarily large.

Theorem 9. Let $G$ be a connected graph of order $n$ and size $m$. Then the following hold:

(i) $h^{\prime}(G) \geqslant \sqrt{\frac{2 m(2 m-n)}{n}}$.

(ii) If $G \neq K_{2}$, then $h^{\prime}(G) \geqslant \sqrt{2 m}$.

Proof. (i) Let $d_{1}, \ldots, d_{n}$ be the degree sequence of $G$. Then by the Cauchy-Schwartz inequality we have

$$
\left(\begin{array}{c}
h^{\prime}(G) \\
2
\end{array}\right) \geqslant|E(L(G))|=\sum_{i=1}^{n}\left(\begin{array}{c}
d_{i} \\
2
\end{array}\right) \geqslant \frac{\left(\sum_{i=1}^{n} d_{i}\right)^{2}}{2 n}-m .
$$

Thus $\frac{h^{\prime}(G)^{2}}{2} \geqslant\left(\begin{array}{c}h^{\prime}(G) \\ 2\end{array}\right) \geqslant \frac{2 m^{2}-m n}{n}$ and the proof is complete.

(ii) Since $G$ is connected, $L(G)$ is connected and has $m$ vertices, hence $L(G)$ has at least $m-1$ edges. Therefore

$$
\left(\begin{array}{c}
h^{\prime}(G) \\
2
\end{array}\right) \geqslant m-1
$$

If $G=K_{1}$, then the assertion is clear. Now, assume that $|V(G)| \geqslant 3$. Thus $h^{\prime}(G) \geqslant 2$ and this implies that

$$
\frac{h^{\prime}(G)^{2}}{2} \geqslant m-1+\frac{h^{\prime}(G)}{2} \geqslant m .
$$

Hence $h^{\prime}(G) \geqslant \sqrt{2 m}$.

Theorem 10. Let $G$ be a k-regular graph of order $n$. Then

$$
\left\lceil\frac{1+\sqrt{1+4 n\left(k^{2}-k\right)}}{2}\right\rceil \leqslant h^{\prime}(G) \leqslant 4(k-1) \sqrt{\frac{n k}{2}-1} .
$$

Proof. Since $G$ is a $k$-regular graph, we find that $|E(L(G))|=n\left(\begin{array}{c}k \\ 2\end{array}\right)$. We know that $\left(\begin{array}{c}h^{\prime}(G) \\ 2\end{array}\right)=\left(\begin{array}{c}h(L(G)) \\ 2\end{array}\right) \geqslant|E(L(G))|$. This implies that $\left(h^{\prime}(G)\right)^{2}-h^{\prime}(G)-n\left(k^{2}-k\right) \geqslant 0$. Therefore, we find $h^{\prime}(G) \geqslant\left\lceil\frac{1+\sqrt{1+4 n\left(k^{2}-k\right)}}{2}\right\rceil$. By a result in $[6], h(G) \leqslant 2 \Delta \sqrt{n-1}$. Thus

$$
h^{\prime}(G) \leqslant(4 k-4) \sqrt{\frac{n k}{2}-1} .
$$


Now, we wish to determine the harmonious edge colouring number of the Petersen graph. Let $P$ denote the Petersen graph. By Theorem 10, we have $h^{\prime}(P) \geqslant 9$. We show that $h^{\prime}(P) \neq 9$. We know that $L(P)$ has 15 vertices and 30 edges. Suppose $h^{\prime}(P)=9$, then there are at least 2 colours which are used once, since if there is exactly one colour which is used once, then we should have at least $2 \times 8+1=17$ vertices in $L(P)$, a contradiction. Thus there are two colours say, $i$ and $j$, which are used once. Since $L(P)$ is a 4-regular graph, there are exactly 4 pairs containing $i$ which do not appear on the edges of $L(P)$. The same holds for $j$. So there are at least 7 pairs containing $i$ or $j$ such that do not appear on the edges of $P$. Since there are $\left(\begin{array}{l}9 \\ 2\end{array}\right)=36$ pairs of colours and $|E(L(P))|=30$, at most 6 pairs cannot be appeared on the edges of $L(P)$, a contradiction. So $h^{\prime}(P) \geqslant 10$. But the following shows a harmonious edge-colouring for Petersen graph using 10 colours.

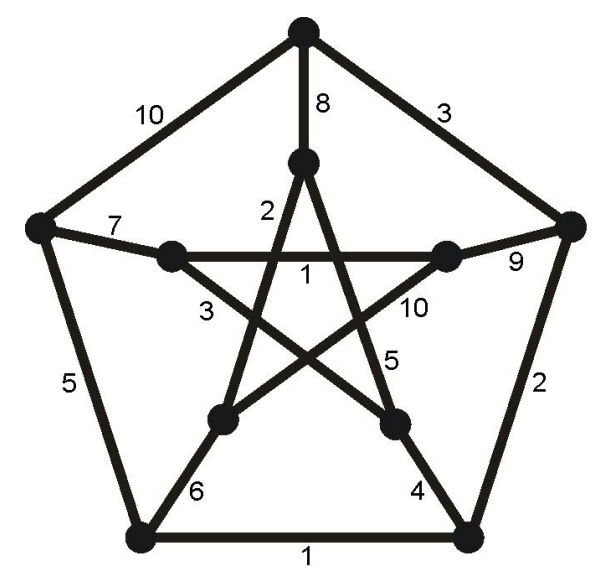

Figure 1: A harmonious edge-colouring of Petersen graph using 10 colours

Acknowledgments. The second author is indebted to the School of Mathematics, Institute for Research in Fundamental Sciences (IPM) for support. The research of the second author was in part supported by a grant from IPM (No. 90050212).

\section{References}

[1] K. Edwards, The harmonious chromatic number of bounded degree trees, Combin. Probab. Comput. 5 (1996), 15-28.

[2] K.J.Edwards, The harmonious chromatic number and the achromatic number, In: R.A.Bailey, ed., Surveys in Combinatorics 1997 (Invited papers for 16th British Combinatorial Conference) (Cambridge University Press, Cambridge, 1997) 13-47.

[3] K. Edwards and C. McDiarmid, The complexity of harmonious colouring for trees, Discrete Appl. Math. 57 (1995), 133-144.

[4] O. Frank, F. Harary and M. Plantholt, The line-distinguishing chromatic number of a graph, Ars Combin. 14 (1982) 241-252. 
[5] J. Hopcroft and M.S. Krishnamoorthy, On the harmonious colorings of graphs, SIAM J. Alg. Disc. Math. 4 (1983) 306-311.

[6] C. J. H. McDiarmid and L. Xinhua, Upper bound for harmonious colorings, J. Graph Theory, 15(1991) 629-636.

[7] B. Wilson, Line Distinguishing and Harmonious Colourings, Graph Colouring, (eds. R. Nelson and R. J. Wilson) Pitman Research Notes in Mathematics 218, Longman Scientific and Technical, Essex (1990) 115-133. 